\section{Eccentric Training: A Load of Interest}

\section{Lorraine R Brilla*}

Department of Health and Human Development, Western Washington University, USA

*Corresponding author: Lorraine R. Brilla, Professor, Department of Health and Human Development, Western Washington University, Bellingham, WA 98225-9067, USA, Tel: 360-650-3056; Fax: 360-650-7447; E-mail: Lorrie.Brilla@wwu.edu

Rec date: May 01, 2015, Acc date: May 07, 2015, Pub date: May 11, 2015

Copyright: (C) 2015 Brilla LR. This is an open-access article distributed under the terms of the Creative Commons Attribution License, which permits unrestricted use, distribution, and reproduction in any medium, provided the original author and source are credited.

\section{Introduction}

Eccentric Muscle Action (EMA) is a critical contributor to training, whether it is for competition or health-related physical performance, such as falls prevention in older adults. EMA is not well explained by the cross bridge contributions and capitalizes on the accumulation of elastic energy during sarcomere lengthening [1]. During lengthening, sarcomere stiffness is reduced, allowing implementation of the series elastic element while storing elastic energy. Roberts and Azizi [2] refer to "springy tissues" and their capacity of stretching while storing elastic strain energy and recoiling later to release energy, when force is reduced. They asserted that tendons have three times the energy storage capacity of muscle fascicles and described tendons as mechanical buffers [3].

The spring structures within muscle include titin, which assists with axial alignment during muscle actions while providing stability to adjacent sarcomeres [4]. Titin elongates working as a molecular spring while accumulating passive energy under active stretch. As actin binds with titin, the free spring length is shortened, increasing titin's stiffness thus enhancing its elastic force accumulation capabilities, while improving force production during EMA [5].

Force is transmitted radially and not only longitudinally as it is sensed across cellular structures with the aid of integrins [6]. Integrins serve as transmembrane receptors sensitive to chemical and mechanical status of the extracellular matrix (ECM). Integrins connect actin filaments with ECM fibers transmitting forces through a clutching system. The ECM supports most of the passive loads, which suggests increased stiffness and limited range of motion could be a direct measure of ECM status [7].

These additional capacities for development of passive force to be combined with active force of cross bridges, EMA is an essential contributor to total movement force. Athletes have capitalized on eccentric training for quite some years, including augmented eccentric loading (AEL) through maneuvers that require apparatus such as elastic bands, medicine balls, or sand-filled weights. EMA strength is retained at greater capacity than concentric contractile force, indicating that this training may be harnessed by older adults to maintain functionality.

\section{Eccentric Muscle Actions in Competition Training}

Recently, an eccentric resistance training and muscle adaptation, with specific focus on hypertrophy, has been reviewed [8]. That source is a comprehensive discussion of eccentric training, this providing good foundation for understanding the current status of research on the topic. The National Strength and Conditioning Association presented an educational article for athletes on the practical utilization of AEL [9]
In athletics, AEL has been studied mostly using drop jump exercise. AEL is applied by employing a tensile load during the airborne and eccentric phases of a drop jump. An additional elastic load may be an effective technique for improving exercise intensity without acute effects on the jumping performance and neuromuscular activation level in highly trained athletes $[10,11]$. Power output, net impulse, takeoff velocity, and jump height are the vital factors that may be improved with AEL [12]. Eccentric focused exercise can enhance performance and has been implicated in injury reduction [13].

\section{Repeated Bout Effect}

Eccentric exercise is associated with development of delayed onset muscle soreness. However, eccentric training results in diminishing the intensity of the soreness, which has been termed the repeated bout effect [14]. Multiple studies have examined the mechanism for repeated bout effect. These studies have implicated reduced muscle lengthening is subsequent eccentric exercise [15], extracellular matrix remodeling [16], and changes in electrical pain threshold of fascia $[17,18]$. Repeated bout effect was not influenced by altered muscle activation or hemodynamic changes [19]. The repeated bout effect infers that eccentric exercise training may be used in health related training since the delayed onset muscle soreness is ameliorated with subsequent bouts of eccentric exercise.

\section{Eccentric Muscle Actions in Health Focused Training}

Eccentric training is commonly used in clinical applications, especially for tendonopathies. In addition to rehabilitation roles, eccentric training which includes AEL may be modified for older adults. Diminished muscle power is largely accepted as a main factor in decreased activities of daily living (ADL) impacting an increased risk for falling in older adults. Increased risk of falling may be due to a number of factors which include: progressive declines in concentric muscle actions, loss of sensory motor integration, and age-related sarcopenia [20]. The loss of concentric muscle actions is greater than eccentric muscle actions with aging in older adults [21]. Most importantly, eccentric muscle force output is retained in older adults which may help to prevent falls because of the brake-like function of eccentric muscle actions [22]. Therefore, eccentric exercise training may enhance muscle strength power output in older adults is important for assessing the risk of falling.

\section{Conclusion}

Eccentric exercise training, including AEL, is an important element in training. Athletes have used various types of AEL with successful increases in power performance. For other applications such as prescribing a fall prevention program, it is important to design the exercises to match functional movements of daily living for older 
Page 2 of 2

adults. It is also vital that the exercises are done in such a manner that movement from the slow loaded eccentric phase to the quick unloaded concentric phase is a fluid pattern. Further research is needed in longterm AEL training for different applications related to sports and health, across various ages. Rehabilitation applications of AEL is an under-studied area, as well as the development of AEL training programs in prevention of injuries, whether it be for athletes or older adults who may be susceptible to falls.

\section{References}

1. Joumaa V, Leonard TR, Herzog W (2008) Residual force enhancement in myofibrils and sarcomeres. Proc Biol Sci 275: 1411-1419.

2. Roberts TJ, Azizi E (2010) The series-elastic shock absorber: tendons attenuate muscle power during eccentric actions. J Appl Physiol (1985) 109: 396-404

3. Roberts TJ, Azizi E (2011) Flexible mechanisms: the diverse roles of biological springs in vertebrate movement. J Exp Biol 214: 353-361.

4. Horowits R, Kempner ES, Bisher ME, Podolsky RJ (1986) A physiological role for titin and nebulin in skeletal muscle. Nature 323: 160-164.

5. Herzog W, Duvall M, Leonard TR (2012) Molecular mechanisms of muscle force regulation: a role for titin? Exerc Sport Sci Rev 40: 50-57.

6. Williams CD, Regnier M, Daniel TL (2012) Elastic energy storage and radial forces in the myofilament lattice depend on sarcomere length. PLoS Comput Biol 8: e1002770.

7. Schwartz MA (2010) Integrins and extracellular matrix in mechanotransduction. Cold Spring Harb Perspect Biol 2: a005066.

8. de Souza-Teixeira F, de Paz JA (2012) Eccentric Resistance Training and Muscle Hypertrophy. J Sport Medic Doping Studies S1: 004.

9. National Strength and Conditioning Association (2014) Practical Utilization of Augmented Eccentric Loading.

10. Aboodarda SJ, Byrne JM, Samson M, Wilson BD, Mokhtar AH, et al. (2014) Does performing drop jumps with additional eccentric loading improve jump performance? J Strength Cond Res 28: 2314-2323.

11. Hrysomallis C (2012) The effectiveness of resisted movement training on sprinting and jumping performance. J Strength Cond Res 26: 299-306.

12. Aboodarda SJ, Yusof A, Abu Osman NA, Thompson MW, Mokhtar AH (2013) Enhanced performance with elastic resistance during the eccentric phase of a countermovement jump. Int J Sports Physiol Perform 8: 181-187.

13. Vogt M, Hoppeler HH (2014) Eccentric exercise: mechanisms and effects when used as training regime or training adjunct. J Appl Physiol (1985) 116: 1446-1454.

14. Paddon-Jones D, Muthalib M, Jenkins D (2000) The effects of a repeated bout of eccentric exercise on indices of muscle damage and delayed onset muscle soreness. J Sci Med Sport 3: 35-43.

15. Yin Lau W, Blazevich AJ, Newton MJ, Xuan Wu SS, Nosaka K (2015) Reduced muscle lengthening during eccentric contractions as a mechanism underpinning the repeated bout effect. Am J Physiol Regul Integr Comp Physiol.

16. Hyldahl RD, Nelson B, Xin L, Welling T, Groscost L, et al. (2015) Extracellular matrix remodeling and its contribution to protective adaptation following lengthening contractions in human muscle. FASEB J. pii: fj.14-266668.

17. Lau WY, Blazevich AJ, Newton MJ, Wu SS, Nosaka K (2015) Changes in electrical pain threshold of fascia and muscle after initial and secondary bouts of elbow flexor eccentric exercise. Eur J Appl Physiol 115: 959-968.

18. Gibson W, Arendt-Nielsen L, Taguchi T, Mizumura K, Graven-Nielsen T (2009) Increased pain from muscle fascia following eccentric exercise: animal and human findings. Exp Brain Res 194: 299-308.

19. Muthalib M, Lee H, Millet GY, Ferrari M, Nosaka K (2011) The repeatedbout effect: influence on biceps brachii oxygenation and myoelectrical activity. J Appl Physiol (1985) 110: 1390-1399.

20. Joshua AM, D'Souza V, Unnikrishnan B, Mithra P, Kamath A, et al. (2014) Effectiveness of progressive resistance strength training versus traditional balance exercise in improving balance among the elderly - a randomised controlled trial. J Clin Diagn Res 8: 98-102.

21. Frontera WR, Hughes VA, Fielding RA, Fiatarone MA, Evans WJ, et al. (2000) Aging of skeletal muscle: a 12-yr longitudinal study. J Appl Physiol (1985) 88: 1321-1326.

22. LaStayo PC, Ewy GA, Pierotti DD, Johns RK, Lindstedt S (2003) The positive effects of negative work: increased muscle strength and decreased fall risk in a frail elderly population. J Gerontol A Biol Sci Med Sci 58: M419-M424. 